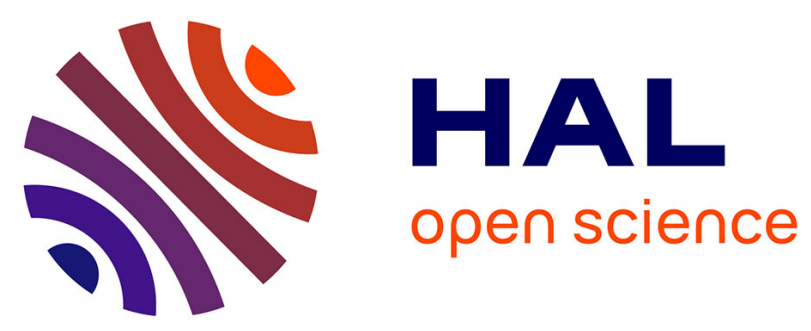

\title{
Generation of Learning Situations According to the Learner's Profile Within a Virtual Environment
}

Kevin Carpentier, Domitile Lourdeaux

\section{To cite this version:}

Kevin Carpentier, Domitile Lourdeaux. Generation of Learning Situations According to the Learner's Profile Within a Virtual Environment. Joaquim Filipe; Ana Fred. Agents and Artificial Intelligence, 5th International Conference, ICAART 2013, Barcelona, Spain, February 15-18, 2013. Revised Selected Papers (449), Springer Berlin Heidelberg, pp.245 - 260, 2014, Communications in Computer and Information Science, 978-3-662-44439-9. 10.1007/978-3-662-44440-5_15 . hal-01096003

\section{HAL Id: hal-01096003 https://hal.science/hal-01096003}

Submitted on 16 Dec 2014

HAL is a multi-disciplinary open access archive for the deposit and dissemination of scientific research documents, whether they are published or not. The documents may come from teaching and research institutions in France or abroad, or from public or private research centers.
L'archive ouverte pluridisciplinaire HAL, est destinée au dépôt et à la diffusion de documents scientifiques de niveau recherche, publiés ou non, émanant des établissements d'enseignement et de recherche français ou étrangers, des laboratoires publics ou privés. 


\title{
Generation of learning situations according to the learner's profile within a virtual environment
}

\author{
Kevin Carpentier and Domitile Lourdeaux \\ Heudiasyc - UMR CNRS 7253, \\ Université de Technologie de Compiègne, 60200 Compiègne, France \\ \{kevin.carpentier, domitile.lourdeaux\}@hds.utc.fr \\ http://www.hds.utc.fr
}

\begin{abstract}
Some working contexts have such a complexity that initial training cannot prepare the workers to handle every kind of situation they migh encounter. This lack of training comes at a high price and leads to productivity loss or low quality manufacturing in industry. Above all, it may be the cause of major accident in high-risk domains. To prevent this risks, virtual environments for training should provide a wide range of learning situations, especially the hard ones, to train the learner how to cope with them. Our purpose is to generate such situations according to the user's capacities. Drawing on the Zone of Proximal Development, we designed a learner's profile based on a multimensional space of classes of situations. Each point of the space depicts a belief on the learner's ability to handle a kind of situation.

abstract environment.
\end{abstract}

Keywords: virtual environment for training, adaptative, knowledge model

\section{Introduction}

Nowadays' working contexts are getting more and more complex, they are composed of a wide range of situations. Training is a major issue in industry for different reasons. It prevents accident in domain where security is critical (high-risk industry, nannies training), it fosters productivity in high-performance industry (aeronautic assembly, submarine maintenance), it also prevents manufacturing defects where customer satisfaction is a key point. Most commonly, in a professional environment, operative attends a short training before getting on the site. They lack of experience and each new situation is difficult to handle because it is a whole new one. It is widely accepted that experience is the most important way to develop professional skills in these domains. By encountering various situations, apprentices may consolidate their knowledge and build their own effective mental representations of the task processing. Moreover, it is accepted that situated learning can offer an efficient learning framework. As such training is expensive and requires the material to be requistionned, virtual environment for training have been proven to be a good solution to provide learning in complex situations [1]. 
By simulating the work context, these environments deliver a wide range of real situations. However, providing content is not enough to ensure an efficient learning. The content must be adapted to the learner's profile and historic : what has been learned? What needs to be learned next? Which errors are most commonly made? Besides, the content answering these questions must be provided in an engaging way. Our goal is to generate pedagogical content adapted to the learner level and presented through a story in which the learner will feel involved. The content proposed must enable learners to meet many and varied kinds of situations and keep their motivation at a high level. To fulfill this requirement, we propose to dynamically generate relevant learning situations with regard of the learner's trace and learning objectives. A relevant learning situation is a set of states of the world that will test a subset of skills and knowledge in a efficient and engaging way. As our works fits in the situated learning theory, we considered that each learner builds his own mental representation in disregard of an elicitation of knowledge and skills. Thus, it makes it difficult to control knowledge acquisition. Another issue is to ensure that the generated content is relevant, which means it fulfills both pedagogical and narrative requirements. This also raised the underlying question about the balance between narrative and motivational factors and pedagogical needs.

SELDON, standing for ScEnario and Learning situations adaptation through Dynamic OrchestratioN, aim to generate and control scenario within a virtual environment. As part of the SELDON model, we propose the TAILOR model to generate a canvas which is a sequence of constraints on the state of the world, called situations, that should be met or prevented to facilitate knowledge learning and skills acquisition. The canvas is then used by the other part of Seldon, a scenario planner, DiRECTOR [2] to constrain the simulation. This paper presents our contribution on activities selection based on belief about learner's aptitudes and pedagogical needs. In section 2 we present how our contribution positions in relation to different approaches on adaptive scenarisation. Then, we introduce the overall process of situation constraints generation and present a detailled method of selection of the constraints depending on pedagogical needs in section 3. Section 4 shows an illustration of this selection through the case of nannies training. Then we will present the perspectives we foresee to extend this work and conclude over the whole contribution.

\section{RELATED WORKS}

Adaptive scenarisation is the process of reacting to user's actions to provide content fitted to their need. In videogames, it might be used to adjust difficulty according to player's level without using typical discrete mode such as "Easy","Hard", etc. With adaptive features, players are always in the flow [3]: the difficulty remains high enough to propose a suitable challenge, yet, players can overcome it so that they do not get bored or frustrated. Such a concept might be used to adapt difficulty in a training session so that learners keep a high level of motivation. The system can propose activities that are always difficult 
enough to challenge the learner but always manageable to prevent frustration and loss of motivation. Our objective is twofold: providing adapted content (1) and presenting this content in such a manner it does not cut the user from the flow and, moreover, motivate him (2).

The adaptation can be made at different levels of granularity. A first approach is to have a global adaptation: a whole scenario has been written [4] or generated [5] and the outcomes of the events were scripted beforehand. This approach allows the building of a scaffolding scenario which present many advantages:

- Pedagogical coherence: the scenario ensures a progressive learning through the session, assistance can be given easily at relevant key points;

- Narrative involvement: it is therefore possible to unfold the event as a story which will involve the learner.

A main drawback is the lack of reactivity of the system. As the whole session has been planned, the system cannot reorient the scenario to adapt to the very current learner's state. The only way to cope with it is to foresee each possible path which can represent a huge amount of work. An opposite approach is to provide reactive adaptation by controling the outcomes of learner's actions. It enables:

- Dynamic adaptation: the system triggers outcomes of learner's actions and provides assistances depending on pedagogical needs.

For example, in the application V3S [6], the triggering of a hasardous matter leak is computed in real-time by HERA [7], an intelligent tutoring system, according to a learner's model.

The simulation where the adaptation takes place can be run with opposite approaches: the controlled approaches versus the emergent approaches.

The controlled approach aims to provide a very efficient learning by orchestrating each part of the simulation: state of the objects, virtual character's behaviours, possibilities of action the learner, etc. It permits:

- Pedagogical control: each element of the simulation serves the scenario and pedagogical needs.

This approach which is used in the Generic Virtual Training [8] helps building pedagogically efficient scenarios but disables the possibility to encounter unintended - though relevant - situations. Moreover, such an approach demands an exhaustive modeling of the world functionment which handicaps the evolutivity of the system. The whole modeling has to be reconsidered to avoid incoherence each time an author adds new contents. Any attempt to interfere with the simulation can cause incoherence for the learner: virtual characters become unpredictible, states of objects changes with no coherent reason. As a result, there is no way to explain a posteriori the unfolding of events. These explanations are critical for the learner to understand causes and consequences of events and 
actions and they can be provided at the end of the session or reviewed by a teacher.

By a clever modelling of small behaviors of the world, emergent approaches allow new situations to arise [9]. It also enables:

- Freedom of action: learners are not framed by the task they are supposed to do, they can experiment and discover the outcomes of their actions;

- Autonomous Virtual Characters: as they are not being controlled by a supervisor, virtual characters maintain their autonomy and their behaviours remain coherent throughout the simulation run.

The issue with emergent approaches is the lack of pedagogical control. The simulation runs itself according to initial parameters and there is no way to orchestrate the events to adapt the simulation to the current learner's state. Each of these approaches has attractive features but none of them fulfills our requirements as explained below.

\section{Proposition}

\subsection{Approach}

Our work aims to provide a relevant adaptation at different level of granularity. At the lowest level, adaptation should modulate the consequences of the actions of the learner, this means an even set of events might have different outcomes depending of the expertise of the learner. Then, the adaptation must work on a middle level basis by producing complex sequences of events leading to a specific learning in a session. Finally, skills development requires a learner to follow a path of different learning situations during different learning sessions, the adaptation should also provide information about the path to follow between different sessions. For this purpose, we will try to adopt a balanced approach which is both global and local.

Besides, learners should have a total freedom and the system must react as it would in reality to help them to develop skills from their mistakes. Technical, organisational and human systems are getting more and more complex in working context. An exhaustive explicitation of each possible scenarios beforehand would result in a combinatorial explosion. To adress the growing comlexity of such systems, we chose to model them through an emergent approach. However, as our purpose is to provide an efficient situated learning, we must ensure that relevant assistances are provided to learners as they would be provided in a working context. Moreover, we need to orchestrate dynamically the course of the training to adapt to current learner state. This can only be achieved by controlling the flow of events to some extent. We need to adopt an emergent approach to model the world but we want to provide pedagogical control over it.

To be able to have both global and local adaptation with pedagogical control over an emergent simulation, we propose to orient dynamically the simulation towards specific situations which are consistant with the current state of the 
world, without breaking the coherence of neither object states nor the behaviour of virtual characters. This is the purpose of SELDON, standing for ScEnario and Learning situation adaptation through Dynamic OrchestratioN), which is a part of the Humans platform described below.

\subsection{HUMANS Framework}

The HUman Models-based Artificial eNvironments Software platform is dedicated to the simulation of virtual environments within complex domains where human factors are critical. HUMANS platform allows high cognitive virtual characters and learners to coexist in a simulation.

HUMANS uses three models which were designed to be informed by domain experts (ergonomists, didacticians,etc.):

- Domain (figure 1) describes the world in a static way, the object, physical or abstract, that exists in the world and the relations between them through an ontology. It also includes a dynamic description: possible actions, the behaviours these actions trigger and events that might occur through rules;

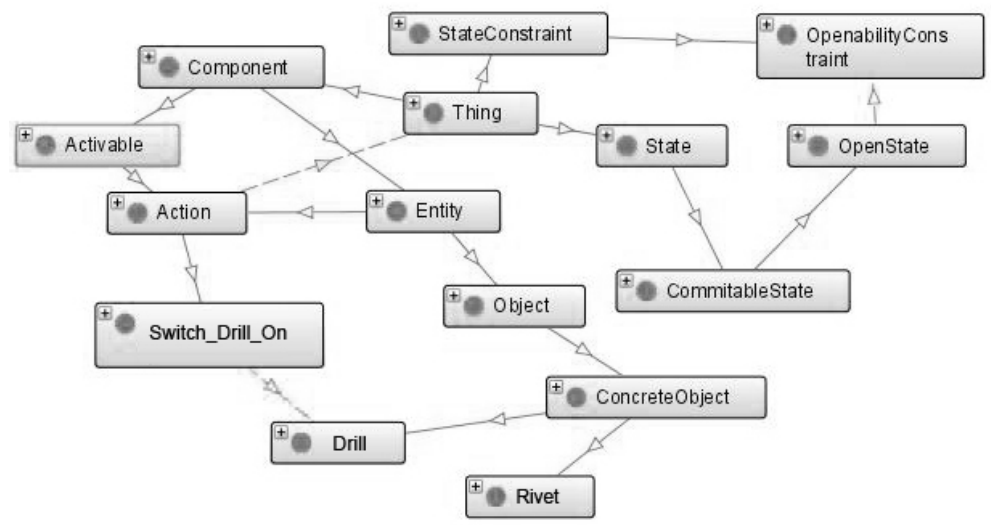

Fig. 1. Part of an ontological representation of DOMAIN

- Activity (figure 2) uses a hierarchical representation of the task to describe the activity as observed on a real site and not as depicted in procedures and protocols. The basic tasks are the actions referenced in DomAIN;

- Causality (figure 3) expresses pertinent causal chains occuring in the environment through a direct acyclic graph. It might describe causal chains of risks (when informed through a risk analysis);

These models manipulate common entities and each unit (Entity in DomaIn, Task in ACTIVITY and Event in CaUSAlity) can be tagged to specify something to which a unit is related (skills, risks, performance criteria.etc.). TAILOR is the first of two parts constituting SELDON. It produces constraints for the second part, DiRECTOR, whose role is to apply this constraints to the simulation. 


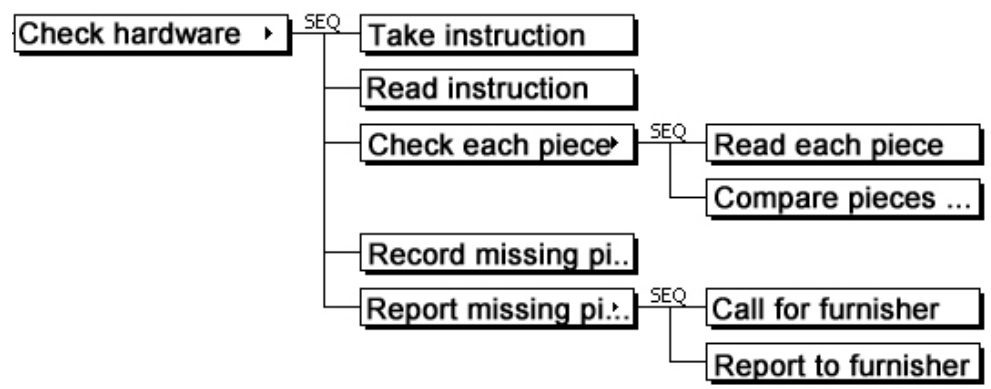

Fig. 2. Hierarchical representation of ACTIVITY

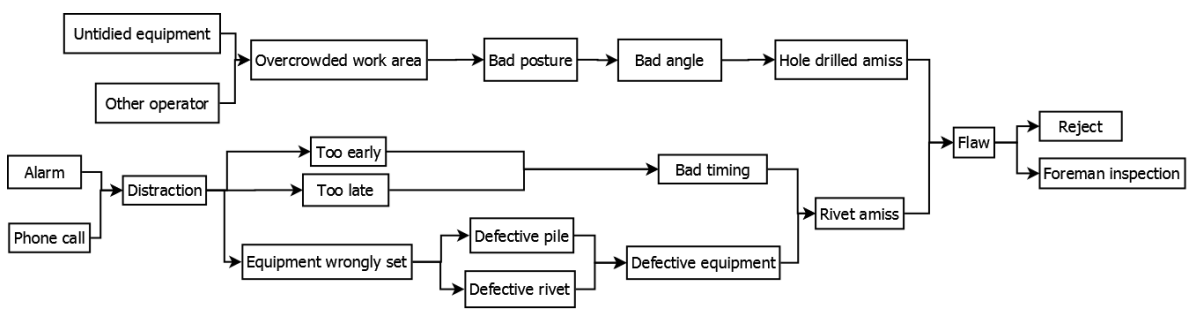

Fig. 3. Graph representation of CAusality

\subsection{General Overview}

As shown in Figure 4, the TAILOR model of constraints generation is divided in three parts: diagnosis, pedagogical selection, narrative framing.

The first part updates a dynamic model based on the Zone Of Proximal Development to establish a diagnosis of learner's capacities.

Second part computes this model to determine a set of situation constraints that fulfill pedagogical needs along with metrics on these situations. They describe if situations shoud be avoided or should be met. Situation constraints describe states of the world which should bring learners to discover/develop/use specific skills and knowledge. One of these situation contraints defines a goal situation toward the simulation should be leading. This situation is not the end of the scenario but merely one of its key points.

In a third part, key points are then framed into common narrative patterns to generate a story and modulate the dramatic tension. The canvas is the succession of situations constraints build upon time. The description of the metrics and of the narrative framing is beyond the scope of this paper.

We present in the following subsection a model for selecting a goal situation according to a uncertain learner's model. 


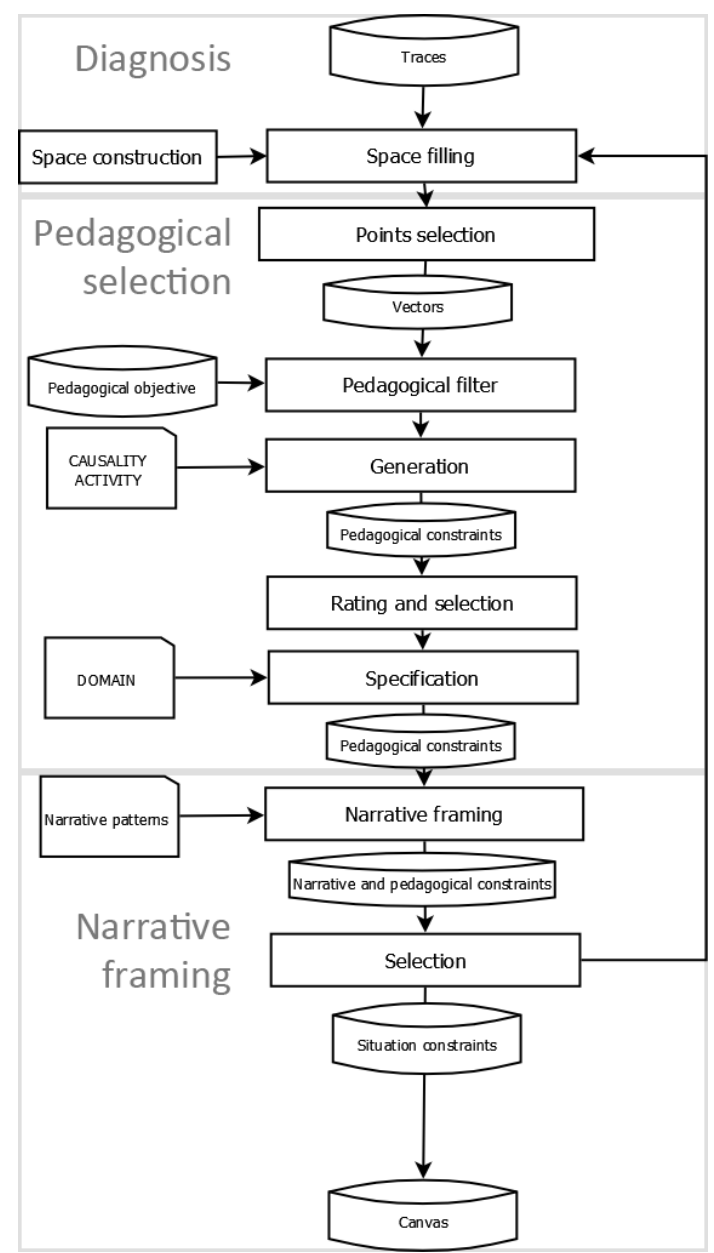

Fig. 4. General Overview

\subsection{Description of the Pedagogical Process}

Input Data. The dynamic mecanism of selection of activities underlying TAILOR lies on both inputs from the learner and the teacher.

- Learner's inputs: each session of training the learner follows is recorded through a trace based on HERA model [7]. As the model is based on activity analysis, traces identify previous situations encountered, errors, causes and consequences, risk produced. Traces are also enriched with activity traces of virtual characters as well as the tracking of causal chains within the environment;

- Teacher's inputs: the teacher can influence the simulation beforehand at different levels: he can select situations which should be encounter during 
the session in the CAUSALITY model, task to be performed in ACTIVITY model and performance criteria to favour.

Diagnosis. On this purpose, the first matter is to establish and maintain a diagnosis of learner's current level of knowledge. As seen in [10], most systems use an elicitation of knowledge, of the influences they have between each other and of the events which are clues of learning.

Actually, we fit our work in the paradigm of situated learning, it would be paradoxal to build a model of skills and knowledge acquisition. Moreover, we want to produce a progressive learning individualized to each learner. Vygotsky proposes the model of Zone Proximal of Development (ZPD) [11] for the context of education in which a student can develop skills inside its confort zone and enlarge it by the help of the teacher. We think the ZPD can be used in a more general learning context than education and that the teacher, which is responsible of the scaffolding, might be played by virtual scaffolding and by an dynamic scenarisation of events. To operationnalize this approach, we choose to deal with a belief the system has in the capabilities of a learner to handle a certain class of situations depicted by constraints. This constraints include all the variable of a situations as observed on site. First, tasks to perform are an obvious variable of situation. Then, constraints are also the cognitive variables which are the parameters to take into account to perform the tasks successfully. We finally choose to add "tags", which are meta-information about task. They help defining if a task is related to some domain-dependant concept such as Quality, Safety, Cleanness, etc. All this variables and there meanings are informed by pedagogical experts of the domain within the models presented in 3.2. These constraints "mold" a space where each situation is described by is component for each variable. Situations encountered by the learner are reported with a belief on whether the he/she handled it successfully or unsucessfully. This space is designed so that two points in space are representative of semantically close situations in the simulation. Beliefs are propagated around each point to estimate a belief on the capabilities of a learner to handle a situation matching another set of constraints semantically close. The Transferable Belief Model and the conjunctive rule of combination (CRC) described in [12] are used to represent and udpate these beliefs.

For each point of this space, describing a class of situations, we have four values:

- a - Belief on the ability to handle this situation,

- $\mathrm{d}$ - Belief on the disability to handle this situation,

- i - Ignorance, either ability or disability

- c - Conflict between belief of ability and disability

With $a+d+i+c=1$

TAILOR parses traces produced by the trace-based system called MONITOR that exists within the HUMANS framework. Based on ACTIVITY and CAUSALITY models, MONITOR aims to record every action agents makes whether they are real learners or virtual characters. These actions are linked to task and high-level 
tasks in the activity hierarchy and are associated to a potentiality to trigger an error, a risk or affecting a performance criteria. Each trace is used as a source of information to update the beliefs about a class of situations. New values are compute according to the application of the conjunctive rule of combination as shown in $1,2,3,4$.

$$
\begin{gathered}
a_{\text {new }}=a_{\text {cur }} * a_{\text {source }}+i_{\text {cur }} * a_{\text {source }}+i_{\text {source }} * a_{\text {cur }} \\
d_{\text {new }}=d_{\text {cur }} * d_{\text {source }}+i_{\text {cur }} r * d_{\text {source }}+i_{\text {source }} * d_{\text {cur }} \\
i_{\text {new }}=i_{\text {cur }} * i_{\text {source }} \\
c_{\text {new }}=1-h_{\text {new }}-d_{\text {new }}-i_{\text {new }}
\end{gathered}
$$

Where $h_{\text {cur }}, d_{\text {cur }}, i_{\text {cur }}$ are the current values, $h_{\text {source }}, d_{\text {source }}, i_{\text {source }}$ are values provided by the trace and $h_{\text {new }}, d_{\text {new }}, i_{\text {new }}, c_{\text {new }}$ are the updated values.

The association between beliefs and the multidimensionnal space described above draws our ZPD we call zpd-space.

\subsection{Pedagogical Selection of Activities.}

As the learner progresses throughout activities and sessions, the space is filled with points and associated beliefs are updated thanks to the monitoring module.

TAILOR will then select a set of points in this space to generate a new situation. The difficulty lies in determining which points will produce an efficient learning. The selection is made based on the 4 values aforementionned using pedagogical rules.

- Points where belief has a high ignorance-value are not likely to be interesting;

- Points where belief has a high ability-value are not interesting to produce new learning, but they can be used in the beginning of the session to make the learner at ease;

- Points where belief has a high disability-value are interesting, because they are the proof of an error, a violation and more generally a misconception. Depending on specific pedagogical rules, the situation will be avoided or, on the contrary, a learning situation will be generate to break the misconception through an assistance;

- Points where belief has a high conflict-value are interesting. Mathematically, it means the different sources of information are contradictory. In our case, it means the learner is able to handle a situation in a specific context but a misconception prevent him from using the same skills in another context.

A set of pedagogical rules helps selecting relevant points according to these values and pedagogical objectives. After this filtering, TAILOR compute DOMAIN, ACtivity and CAUsality model to determine which events and which activities responds to these constraints. 
Output Data. At each iteration, TAILOR generates a set of situation constraints associated to a desirability which represent how desired this state of the world is (see Table 1). A negative value describe a situation that should be avoid. One situation is tagged as the goal situation. A situation is depicted by a subset of triple describing a specific state of the world using the formalism of DOMAIN.

Table 1. Situations and desirability.

\begin{tabular}{|c|c|}
\hline Situation constraints & Desirability \\
\hline Sit $_{1}$ & $D_{1} \in[-1,1]$ \\
\hline$\ldots$ & $\ldots$ \\
\hline Sit $_{n}$ & $D_{n} \in[-1,1]$ \\
\hline Sit $_{\text {Goal }}$ & 1 \\
\hline
\end{tabular}

\section{Example: Training in aeronautics assembly}

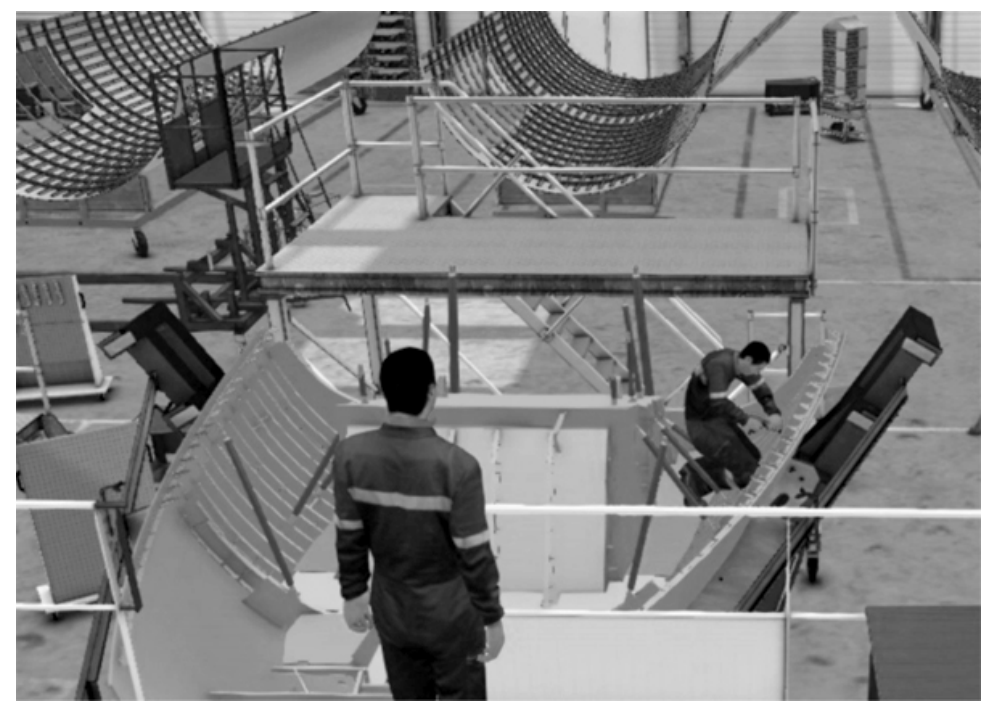

Fig. 5. A screenshot of NIKITA - Aeronautics training simulator

For the purpose of the NIKITA (Natural Interaction, Knowledge and Immersion in Training for Aeronautics) we applied the model generation to a scenario of airplane assembly operative training. The LATI lab of Paris-Descartes University made a torough study of the work environment in Méaulte (Picardy, France) facility of the AEROLIA group. Their analyses, video and comments 
made possible the authoring of the DomAIn model and the ACTIVITY tree of the operatives. The Méaulte facility capabilities are mainly the assembly of the nose fuselage and of the lower shell fuselage. Those fuselage has been made of composite panel since 2010. The NIKITA application aims to train the operative to perform riveting task on various material. The LATI Lab runs an analysis based on conceptual structure for the representation [13]. We use this information to design a zpd-space. For the sake of the example, we will only focus on two dimensions of the zpd-space. The first dimension we consider, named $M a-$ terial, depicts the different materials on which the learner has to work. These include common alloy but also composite material which requires special care. The second dimension, named Time Pressure, depicts the level of time which is set upon the operative to perform his work. The pedagogical objective has arbitrarily been set to Material = Composite. In the following example M stand for the Material axis and TPL for the Time Pressure Level axis.

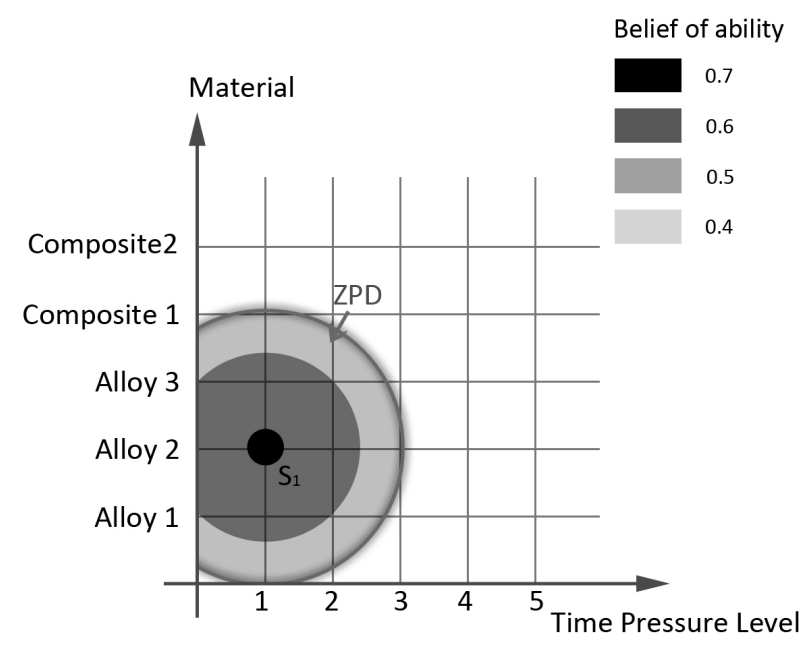

Fig. 6. ZPD-space initialisation in aeronautics training simulator

Step 1 : Initialisation. In this example we consider there is no conflict, and that the learner has successfully handled situation $S_{1}$ (figure 6). The darker the color the surer the system knows that the learner will be able to handle a situation generated from this point. Blank areas express the lack ok knowledge about the learners habilities. The ZPD is where the belief on the ability of the learner is beyond a threshold ( 0.4 in this case).

Step 2. In regard of the pedagogical objectives, which here emphasize on $M=$ Composite, the system selects points to privilege this kind of situation. The point 


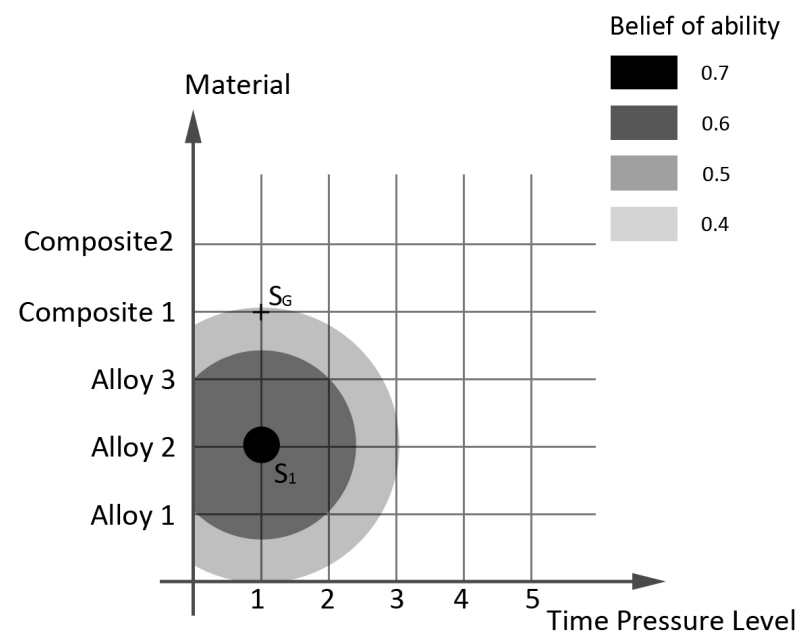

Fig. 7. Situations generation

( $M=$ Composite $1, T P L=1$ ) is selected (figure 7 ). By computing the ACTIVITY model, TAILOR determines tasks requiring a $(M=$ Composite $1, T P L=1)$ set of skills. The task "Drilling the front bark on the A3YY" is an activity that fulfills this requirement. In regard with this, the scene is initialized with a bark of an A3YY where the learner would be asked to fix a support on the bark.

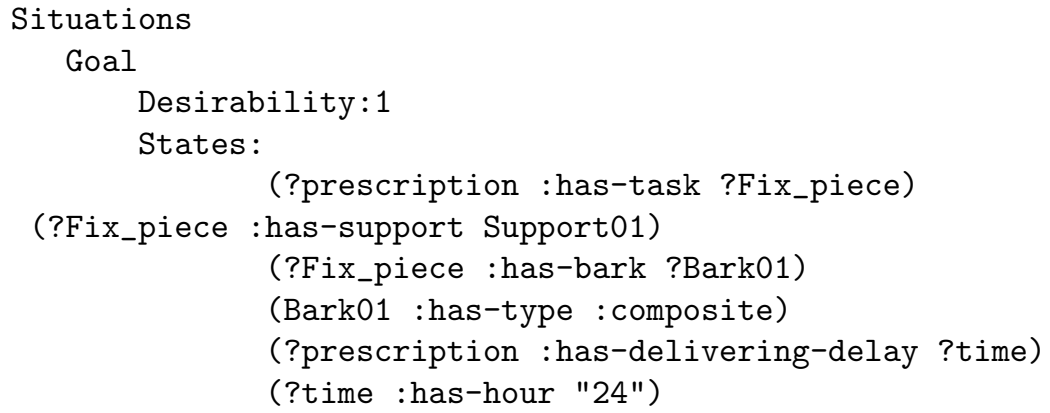

Step 3. The learner reacted well to the previously generated situation by making good quality drilling and taking into account specificities of composite material, the ZPD-space is updated. The point $S G(M=$ Composite1, $T P L=3)$ is now selected (figure 8)., the system has a belief of 0.4 for the learner to handle this class of situations. This class of situations will allow the evaluation of the capacities of the learner to work with composite material with a time pressure. By computing the ACTIVITY model, TAILOR determines that time pressure is induced when the delivering delay is reduces and the foreman ask for a quicker delivering (which is an action called AskForHaste ). But, a "rush" in work may 


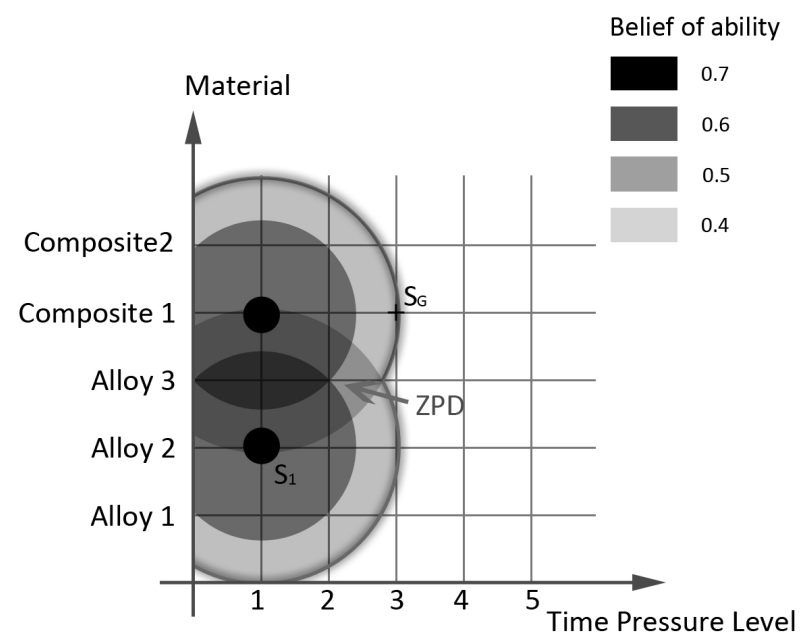

Fig. 8. ZPD-space udpate and new activities selection

cause an overcrowded workspace by virtual agent which will react to the new state of the world, yet this is not the kind of situation that has to be encountered. TAILOR generate another constraints to keep the crowd level in workspace low.

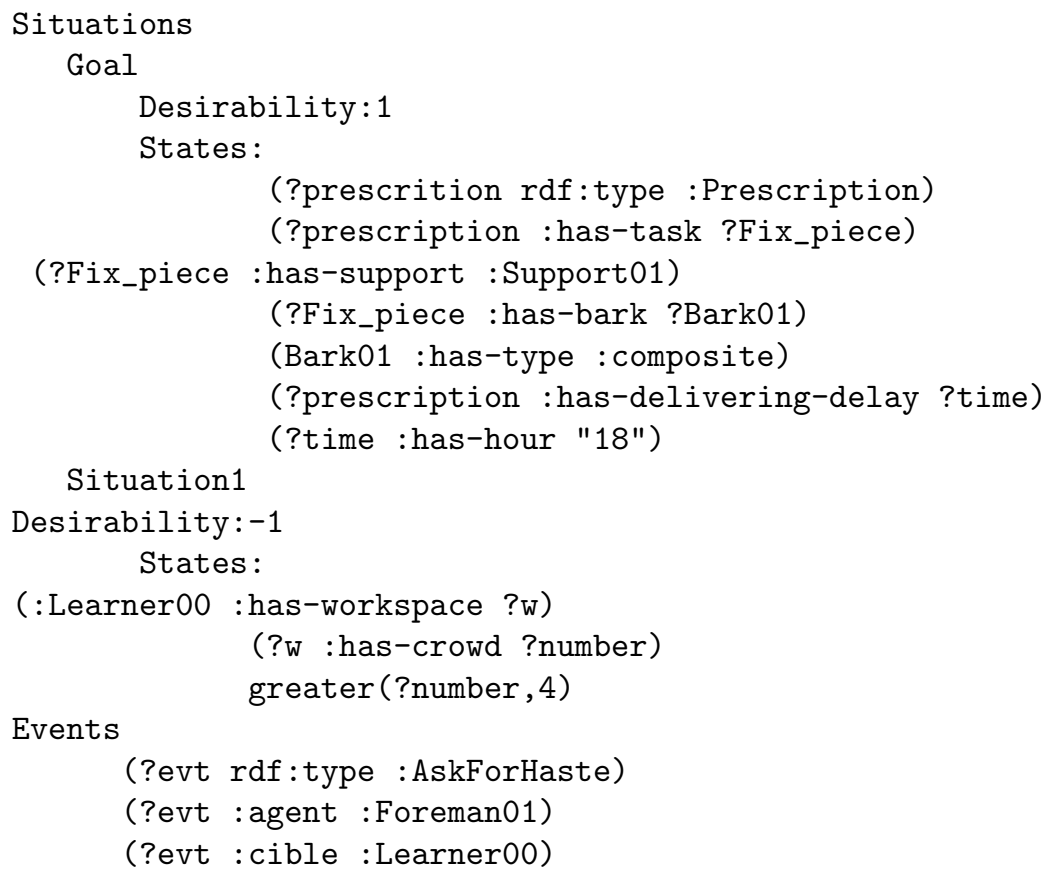




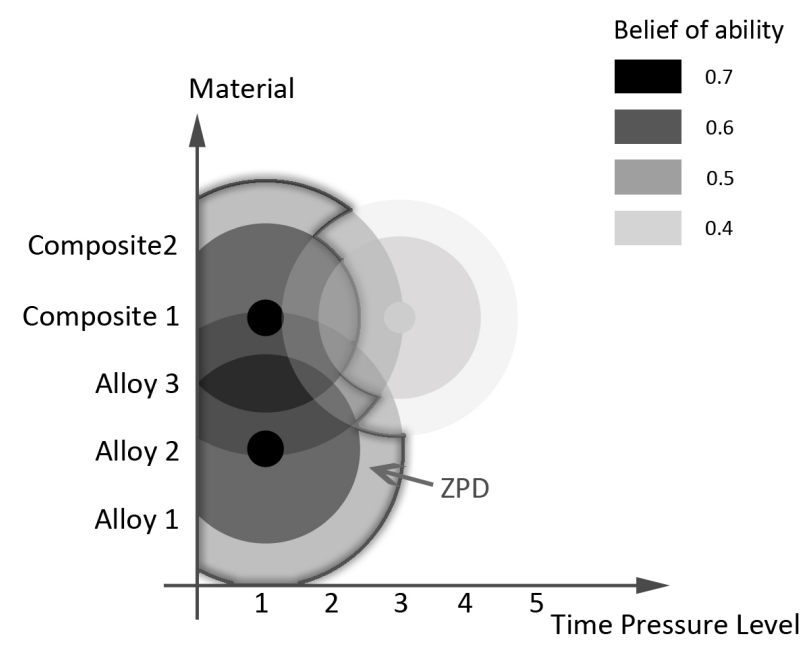

Fig. 9. End of training session

Final Step. In order to deal with the time pressure, the learner choose not to do some specific tasks. These task are allowed not to be done when handling common material. But, these task are mandatory when handling composite material. This shows a lack in the knowledge of the learner. The ZPD-space is updated accordingly (figure 9 ).. In order to prevent future mistake, TAILOR propose to trigger crack in the panel. Thus, the learner will likely understand that more care were needed. In the ZPD-Space, the information of a remediation has been recorded for this class of situations ( $M=$ Composite $1, T P L=3)$. The effectiveness of the remediation will be tested in a further training session.

\section{$5 \quad$ Perspectives and future works}

The ZPD-space we are currently using are designed by-hand according to the knowledge models informed by didacticians and ergonomists, i.e, we choose the axis, their scale and their meaning. Future works will explore how to generate those axis automatically by parsing our knowledge models. The issue is that some variables migh correlated which may add a flow on our process. We may use data analysis method such as principal component analysis [14] to build a new space with uncorrelated variables.

Selection of activities is the first part of our work. To provide adapted content is essential but to involve the learner, we need to use motivational factors. Modulating the dramatic tension is a possible solution. To create tension, a story must be built upon the events and the world depicted within the simulation. The aim is to provide an interest for the learner by showing the virtual characters as story characters who can be helpers or opponents, the events as plot points that will increase or decrease the tension. We plan to use narrative pattern, as described 
by [15], [16] or [17] to extends current pedagogicial situations. The element described by the situations will be fitted with element from a pattern such as location, helpers, opponents, goal,etc. For one pedagogical situation, many narrative configurations are possible. We will use a measure of the narrative utility based on earlier events in the simulation. The utility will maximize if it furthers the development of the story depicted in past events without disrupting the whole coherence.

\section{Conclusions}

We proposed in this paper a model to dynamically generate scenarios in a virtual environment regarding learner's capacities. The process uses a phase of diagnosis which compute traces at the initialisation and in real time. It operationnalizes the theory of zone of proximal development through a multidimensionnal space of beliefs, updated at each task performed by the learner within the virtual environment. Then the system computes current world state to determine which situation can take place to answer ZPD and pedagogical objectives requirements. We build a first prototype within the Humans platform working on the example of aeronautics assembly. Our future works will focus in the narratives consideration by framing the successive situations in a narrative pattern to relate a story. Besides, we are exploring new method to build the zpd-space.

\section{Acknowledgements}

This work is part of the ANR project: NIKITA (Natural Interaction, Knowledge and Immersive systems for Training in Aeronautic). Partners are: Heudiasyc, Paris Descartes University, CEA-LIST, Emissive, EADS, AEROLIA and the technical school Henry Potez. We want to thank Catherine Delgoulet and Vincent Boccara from the LATI for their torough analysis which enable us to build our use-case.

\section{References}

1. Amokrane, K. and Lourdeaux, D. (2009). Virtual Reality Contribution to Training and Risk Prevention. In Proceedings of the 2009 International Conference on Artificial Intelligence (2009)

2. Barot, C., Lourdeaux, D., and Lenne, D.: Dynamic Scenario Adaptation Balancing control, Coherence and Emergence. In Proceedings of the 5th International Conference on Agents and Artificial Intelligence (2013)

3. Csikszentmihalyi, M.: Flow: The Psychology of Optimal Experience. Harper Perennial (1991)

4. Marion, N.: Modélisation de Scénarios Pédagogiques pour les Environnements de Réalité Virtuelle d'Apprentissage Humain, PhD thesis, Université de Bretagne Occidentale (2010) 
5. Niehaus, J. M., Li, B., and Riedl, M. O.: Automated Scenario Adaptation in Support of Intelligent Tutoring Systems. In Proceedings of the 24th Conference of the Florida Artificial Intelligence Research Society, Palm Beach, Florida (2011)

6. Barot, C., Burkhardt, J.-M., Lourdeaux, D., and Lenne, D.: V3S, a Virtual Environment for Risk Management Training. In JVRC11: Joint Virtual Reality Conference of EGVE - EuroVR, Nottingham, (2011)

7. Amokrane, K. and Lourdeaux, D.: Pedagogical System in vVirtual Environment for High-Risk Sites. In Proceedings of the 2nd International Conference on Agents and Artificial Intelligence (2010)

8. Gerbaud, S., Mollet, N., Ganier, F., Arnaldi, B., and Tisseau, J.: GVT: a Platform to Create Virtual Environments for Procedural Training, In IEEE Virtual Reality, pp. 225-232. IEEE Press, New York (2008)

9. Shawver, D.: Virtual Actors and Avatars in a Flexible User-determined-scenario Environment. In Virtual Reality Annual International Symposium (1997)

10. Brusilovsky, P., and Millan, E.: User Models for Adaptive Hypermedia and Adaptive Educational Systems, Lecture Notes in Computer Science (2007)

11. Vygotsky, L. S.: Mind in Society. Harvard University Press, Cambridge (1978)

12. Smets, P. and Kennes, R.: The Transferable Belief Model. Artificial Intelligence 66, $191-234$ (1994)

13. Pastré, P.: La Conceptualisation dans l'Action : Bilan et Nouvelles Perspectives, Éducation permanente, 139, pp. 13-35 (1999)

14. Pearson, K.: On Lines and Planes of Closest Fit to Systems of Points in Space, Philosophical Magazine 2 (11), pp. 559-572 (1901)

15. Campbell, J.: The Hero With a Thousand Faces, New World Library (2008)

16. Propp, V. I.: Morphology of the Folktale, University of Texas Press (1968)

17. Greimas, A. J.: Sémantique structurale: recherche et méthode, Larousse edition (1966) 